ARTICLE

\title{
Dynamic upconversion multicolour editing enabled by molecule-assisted opto-electrochemical modulation
}

\author{
Yiming Wu (1) ${ }^{1}$, Jiahui Xu (10 ${ }^{1}$, Xian Qin (10 ${ }^{1 凶}$, Jun $X u^{1,2} \&$ Xiaogang Liu (1) 1,2,3,4凶
}

Controlling nonlinear optical signals electrically offers many opportunities for technological developments. Lanthanide-activated nanoparticles have recently emerged as leading platforms for nonlinear upconversion of infra-red excitation within nanometric volumes. However, manipulation of upconversion emission is restricted to varying percentages of component materials, nanocrystal structure, and optical pumping conditions. Here, we report temporal modulation of anti-Stokes luminescence by coupling upconversion nanoparticles with an electrochemically responsive molecule. By electrically tailoring orbital energy levels of the molecules anchored on nanoparticle surfaces, we demonstrate reversible control of molecular absorption, resulting in dynamic colour editing of anti-Stokes luminescence at single-particle resolution. Moreover, we show that a programmable logic gate array based on opto-electrochemical modulation can be constructed to convert information-encrypted electrical signals into visible patterns with millisecond photonic readout. These findings offer insights into precise control of anti-Stokes luminescence, while enabling a host of applications from low-threshold infrared logic switches to multichannel, high-fidelity photonic circuits.

\footnotetext{
${ }^{1}$ Department of Chemistry, National University of Singapore, Singapore, Singapore. ${ }^{2}$ Center for Functional Materials, National University of Singapore Suzhou Research Institute, Suzhou, China. ${ }^{3}$ Joint School of National University of Singapore and Tianjin University, International Campus of Tianjin University, Fuzhou, China. ${ }^{4}$ Institute of Materials Research and Engineering, A*STAR, Singapore, Singapore. ${ }^{凶}$ email: chmqinx@nus.edu.sg; chmlx@nus.edu.sg
} 
$\mathrm{N}$ onlinear photonic devices capable of detecting and modulating infrared signals as a communication medium are essential for technological developments in thermography, night vision, medical diagnosis, information encryption, and optical communication $^{1-6}$. Such optoelectronic applications can be accomplished by using nonlinear nanocrystals that enable frequency conversion of invisible infrared radiation to visible luminescence. The ability to dynamically control optical functionalities of the upconversion medium by modulating external stimuli, such as temperature, pressure, magnetic or electric field, will facilitate development of multifunctional, nonlinear optoelectronic devices ${ }^{7-9}$. In particular, there is strong demand for electrical control over optical properties of upconversion nanomaterials, as such control will not only allow monolithic integration of photonic elements with optoelectronic functionalities but will also lead to many intriguing phenomena in nonlinear regimes ${ }^{10-12}$.

Compared with conventional nonlinear nanocrystals, lanthanideactivated upconversion nanoparticles (UCNPs) allow more-efficient frequency upconversion owing to abundant, physically existing energy states. UCNPs also exhibit excellent photostability, large anti-stokes shift, low pumping threshold, and broadly tunable multicolour emission ${ }^{13,14}$. Temporal modulation of upconversion emission can be realised by varying excitation wavelengths or by adjusting excitation power densities ${ }^{15-19}$. These methods, however, require multiple excitation sources and stringent control over crystalline phase, chemical composition, and surface ligands. Variations in the thermal field also allow reversible luminescence modulation of UCNPs, though these are impractical for device configuration $^{20,21}$. External stimulus-responsive hosts, such as liquid crystal polymer networks, ferroelectric or optomagnetic materials, have been studied to manipulate emission dynamically under external electrical or magnetic stimulation ${ }^{22-25}$. However, high phonon energy and low upconversion efficiency of these systems, as well as a limited range of emission colours, hinder their practical utility for multicolour switching. Despite enormous efforts, implementation of reversible, dynamic, full-colour emission modulation of UCNPs through electric field stimulation has not been achieved, largely owing to the non-conducting nature of conventional lanthanide-doped host materials and shielding effects of $4 \mathrm{f}$ electrons against external electrostatic perturbation.

In this work, inspired by electrochromic organic molecules for optoelectronic applications, such as rewritable, optical labels, and colour-changing metasurfaces ${ }^{26,27}$, we reasoned that fast, multicolour switching of upconversion luminescence should be possible by electrically controlling energy transfer from UCNPs to electrochemically responsive organic molecules. Viologen molecules are the choice of materials for optical switching owing to their good photostability, fast temporal responses, high absorption coefficients, and tailorable absorption wavelengths, as well as ease of structural modification ${ }^{28-30}$. Dynamic modulation of upconversion emission colour and intensity can be achieved with molecular-assisted surface electrochemical tuning (MASET) (Fig. 1a). When pumped with a $980 \mathrm{~nm}$ laser, UCNPs exhibit steady luminescence. Upon stimulation with an external electric field, viologen molecules receive electrons and convert from an oxidised state to a reduced state via a redox electrochemical process, resulting in electrically controlled energy transfer from UCNPs to viologen molecules owing to spectral overlap between nanocrystal emission and dye absorption. A recovery of upconversion emission can be rapidly achieved through a reversible redox reaction under a reverse electric field (Fig. 1b).

\section{Results}

Electrically switchable upconversion luminescence. We experimentally validated the feasibility of the MASET strategy to achieve electrically switchable luminescence and to investigate the involvement of energy transfer between UCNPs and viologen molecules under electrical gating (Fig. 2a). UCNPs with multicolour emission properties were prepared by doping different lanthanide ions (e.g., $\mathrm{Yb}^{3+}, \mathrm{Er}^{3+}, \mathrm{Tm}^{3+}, \mathrm{Eu}^{3+}$, and $\mathrm{Tb}^{3+}$ ) into specific layers of hexagonal-phase $\mathrm{NaYF}_{4}$ and $\mathrm{NaGdF}_{4}$ host materials $^{31-34}$ (Fig. 2b, c and Supplementary Figs. 1, 2). Owing to the nonconductive properties of the host materials, a layer of $\mathrm{TiO}_{2}$ was coated onto silica-modified UCNPs to yield semiconducting surfaces (Fig. 2d, e and Supplementary Figs. 3-5). In addition, $\mathrm{Ti}^{4+}$ ions possess a strong affinity for highly charged electrochromic molecules ${ }^{35}$. We designed and fabricated a sandwich-like electrochemical cell comprising viologen molecule-modified UCNPs (Fig. 2a). A paste containing $\mathrm{TiO}_{2}-$ coated $\mathrm{NaYF}_{4}: \mathrm{Yb} / \mathrm{Er}$ nanoparticles was deposited on a fluorinedoped tin oxide-coated glass and then calcined to form a semiconducting hybrid film. Under $980 \mathrm{~nm}$ laser excitation, the nanoparticle thin film exhibited green and red emission bands at 540 and $654 \mathrm{~nm}$ (Supplementary Figs. 6-8). By virtue of its excellent optical properties, an organic molecule, 1,1'bis(2-phosphonoethyl)-4,4'-bipyridinium dichloride (PV), was selected and synthesised as the electrically active molecule ${ }^{36}$ (Supplementary Figs. 9, 10).

Under appropriate electric field manipulation, PV molecules displayed reversible transformation between their di-cation oxidised form $\left(\mathrm{PV}^{2+}\right)$ and cation-radical reduced form $\left(\mathrm{PV}^{\cdot+}\right)$ (Fig. 2f). Notably, in contrast to the oxidised state, which is transparent to visible and NIR radiation, the reduced state displays a broad absorption band, overlapping considerably with emission bands of $\mathrm{NaYF}_{4}: \mathrm{Yb} / \mathrm{Er}$ nanoparticles (Fig. $2 \mathrm{~g}$ and Supplementary Fig. 11). Under $980 \mathrm{~nm}$ irradiation, the hybrid device gave rise to dominant yellow emission peaks at 540 and $654 \mathrm{~nm}$, corresponding to ${ }^{2} \mathrm{H}_{11 / 2} \rightarrow{ }^{4} \mathrm{I}_{15 / 2}$ and ${ }^{4} \mathrm{~S}_{3 / 2} \rightarrow{ }^{4} \mathrm{I}_{15 / 2}$ transitions of $\mathrm{Er}^{3+}$ ions. However, yellow emission was significantly suppressed upon applying an electric field of $-3 \mathrm{~V}$. By monitoring voltage-dependent absorbance of PV molecules at $654 \mathrm{~nm}$, we found that electrochemical conversions of PV molecules can be initiated by applying an electric field of $-2 \mathrm{~V}$. The absorption intensity of PV molecules reached its maximum as the applied voltage was set at $-3 \mathrm{~V}$ (Fig. $2 \mathrm{~h}$ and Supplementary Fig. 12). As a result, photoquenching of UCNPs was precisely controlled under an electric field, by which emission intensity gradually decreased with increasing absorbance of PV molecules (Fig. 2h).

The UCNP/PV hybrid system exhibited good durability and fast switching speed with a rise time of $400 \mathrm{~ms}$ and a decay time of $300 \mathrm{~ms}$, which are orders of magnitude faster than those of photochromic molecule-mediated upconversion tuning ${ }^{18,37}$. Moreover, no noticeable degradation was observed after 47 writing/erasing cycles, and the "on" and "off" states for each cycle remained the same (Fig. 2i and Supplementary Fig. 12), indicating reversible and stable electric-switching characteristics with excellent fatigue resistance. On the other hand, lifetime decay curves measured for $\mathrm{NaYF}_{4}: \mathrm{Yb} / \mathrm{Er}$ nanoparticles in electrochemical cells with or without the electric field remained unchanged (Supplementary Fig. 13), revealing that energy transfer from UCNPs to PV molecules is dominated by a radiative reabsorption process instead of nonradiative Förster resonance energy transfer ${ }^{38}$. Our theoretical investigations suggest that the maximum absorption of oxidised PV molecules is assigned to the electronic transition of HOMO $-3 \rightarrow \mathrm{HOMO}+3$ at $272 \mathrm{~nm}$. In comparison, reduced PV molecules exhibit three dominant electronic transitions of $\beta-\mathrm{HOMO}-2 \rightarrow \alpha-\mathrm{HOMO}+1$ at $387 \mathrm{~nm}, \alpha-\mathrm{HOMO} \rightarrow \alpha-\mathrm{HOMO}$ at $521 \mathrm{~nm}$ and $\alpha-\mathrm{HOMO}-2$ $\rightarrow \alpha-\mathrm{HOMO}+1$ at $645 \mathrm{~nm}$ (Fig. $2 \mathrm{j}$ and Table S1), in good agreement with experimental results. 
a

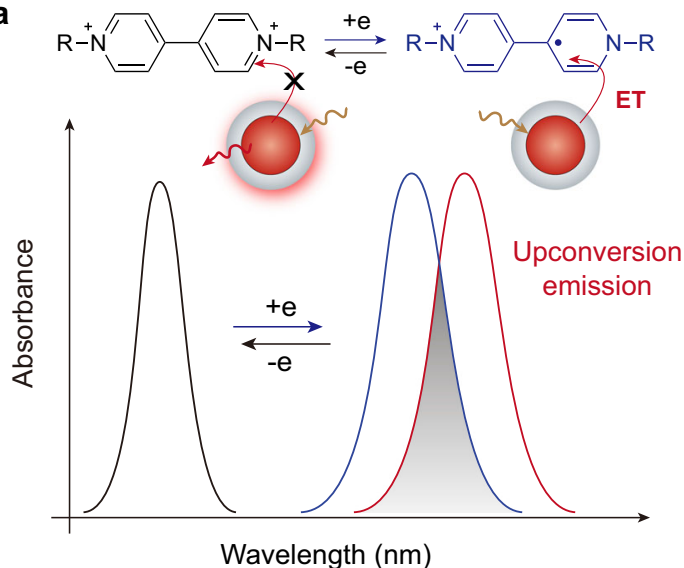

b

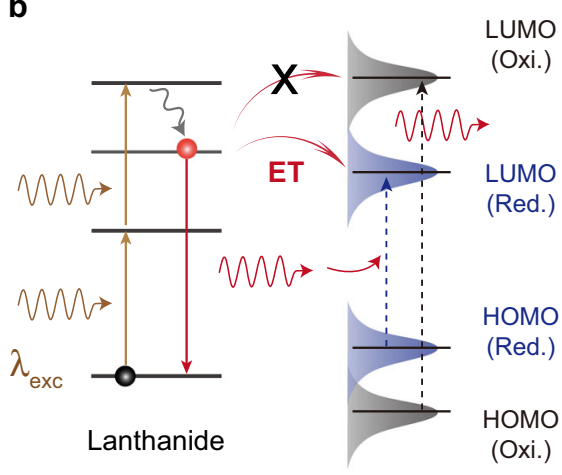

Molecule

Fig. 1 Molecular-assisted surface electrochemical tuning (MASET). a Schematic illustration of electrochemically controlled energy transfer (ET) from UCNPs to viologen molecules ( $R$ represents a functional group). An applied electric field induces electrochemical conversion of viologen molecules. For a given set of nanocrystals and viologen molecules, no energy transfer occurs upon NIR laser excitation $\left(\lambda_{\text {exc }}\right)$, owing to lack of spectral overlap between nanocrystal emission and dye absorption. Applying an external electric field drives the oxidised state (Oxi.) of viologen molecules to the reduced state (red.) through a redox process, resulting in selective quenching of luminescence owing to spectral resonance between nanocrystals and molecules. A reverse electric field oxidises the reduced viologen molecules, leading to a recovery of upconversion emission. $\mathbf{b}$ Simplified energy level scheme of lanthanide-activated UCNPs and schematic energy level diagram of viologen molecules in oxidised (Black) and reduced (Blue) forms, illustrating electrically controlled energy transfer from UCNPs to dye molecules. Solid and dashed lines represent electronic transitions.

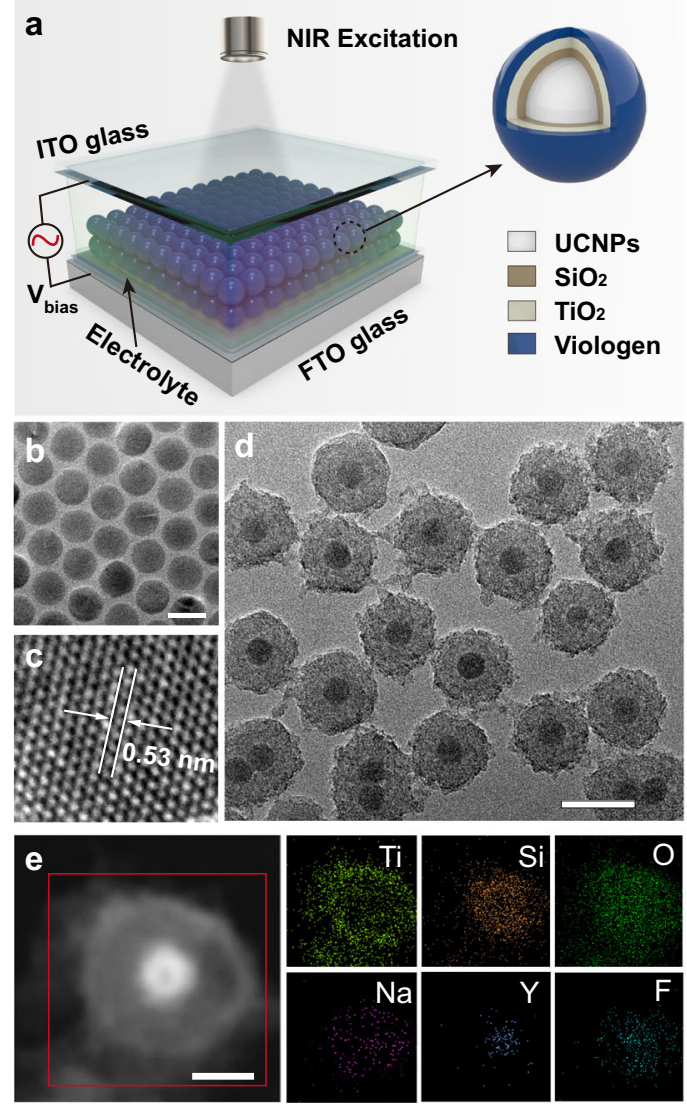

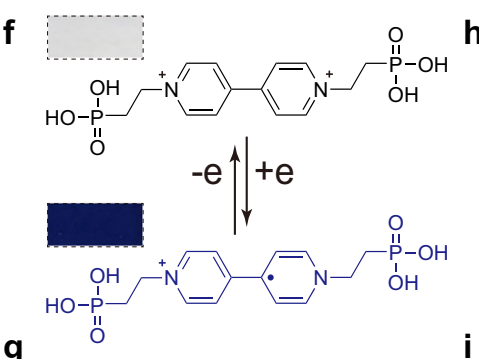

g

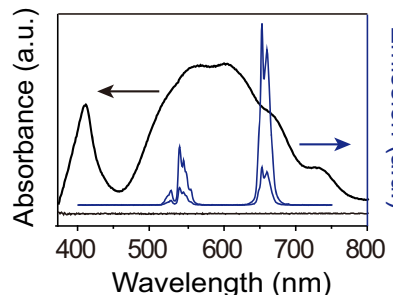

j
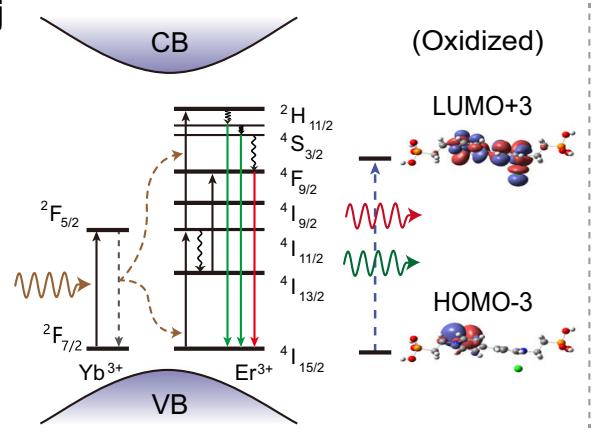

h
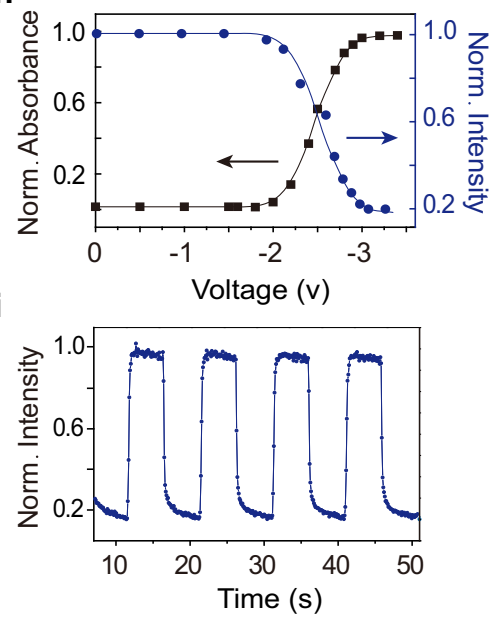

Fig. 2 Experimental demonstration of electrically switchable upconversion luminescence. a Schematic of an electrochemical cell based on viologen molecule-modified UCNPs. b, c Representative TEM images of NaYF 4 :Yb/Er nanoparticles under study. Scale bar: 20 nm. d, e TEM images of NaYF 4 :Yb/ $\mathrm{Er}_{\mathrm{SiO}} @ \mathrm{TiO}_{2}$ nanoparticles and corresponding EDX elemental mapping of a single nanoparticle. Scale bar: $50 \mathrm{~nm}$ for $\mathbf{d}$ and $20 \mathrm{~nm}$ for e. f Chemical structures of 1,1'-bis(2-phosphonoethyl)-4,4'-bipyridinium dichloride (PV) in oxidised and reduced forms. Insets show optical images of the oxidised and reduced PV molecule, respectively. $\mathbf{g}$ Absorption spectra of PV molecules of a hybrid film in oxidised and reduced states (black) and emission spectra of the hybrid film in "bright" and "dark" states (blue). h Normalised absorption intensity of PV molecules of a hybrid film at 654 nm (black) and normalised upconversion emission intensity at $654 \mathrm{~nm}$ (blue) as a function of the applied potential. i Reversible luminescence on/off cycles, recorded at $654 \mathrm{~nm}$ under alternating applied potential $( \pm 3 \mathrm{~V})$. j Simplified energy level diagram of $\mathrm{NaYF}_{4}: \mathrm{Yb} / \mathrm{Er}$ nanoparticles and the proposed mechanism underlying the opto-electrochemical modulation. 
a

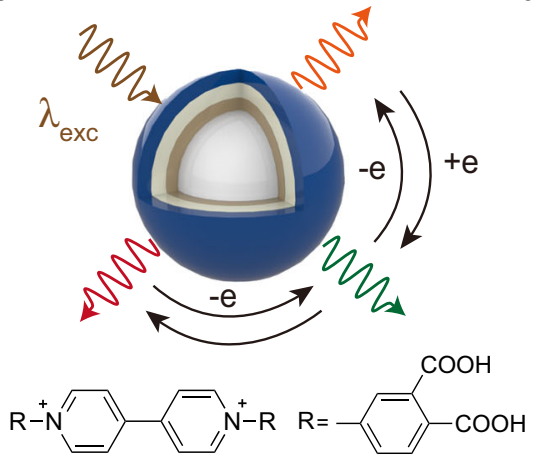

b

d

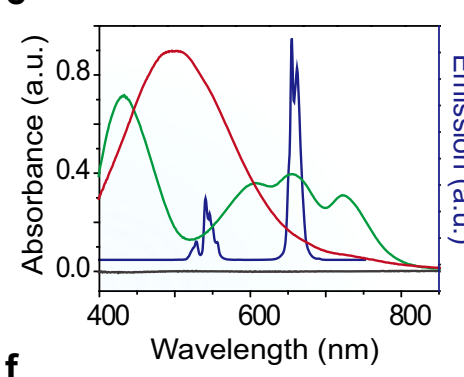

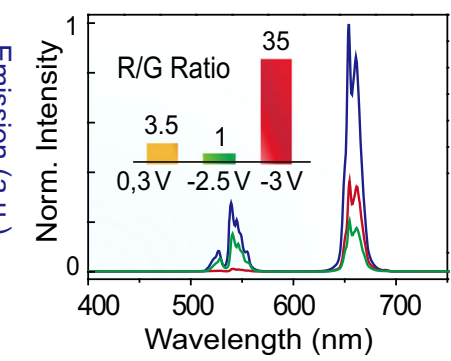
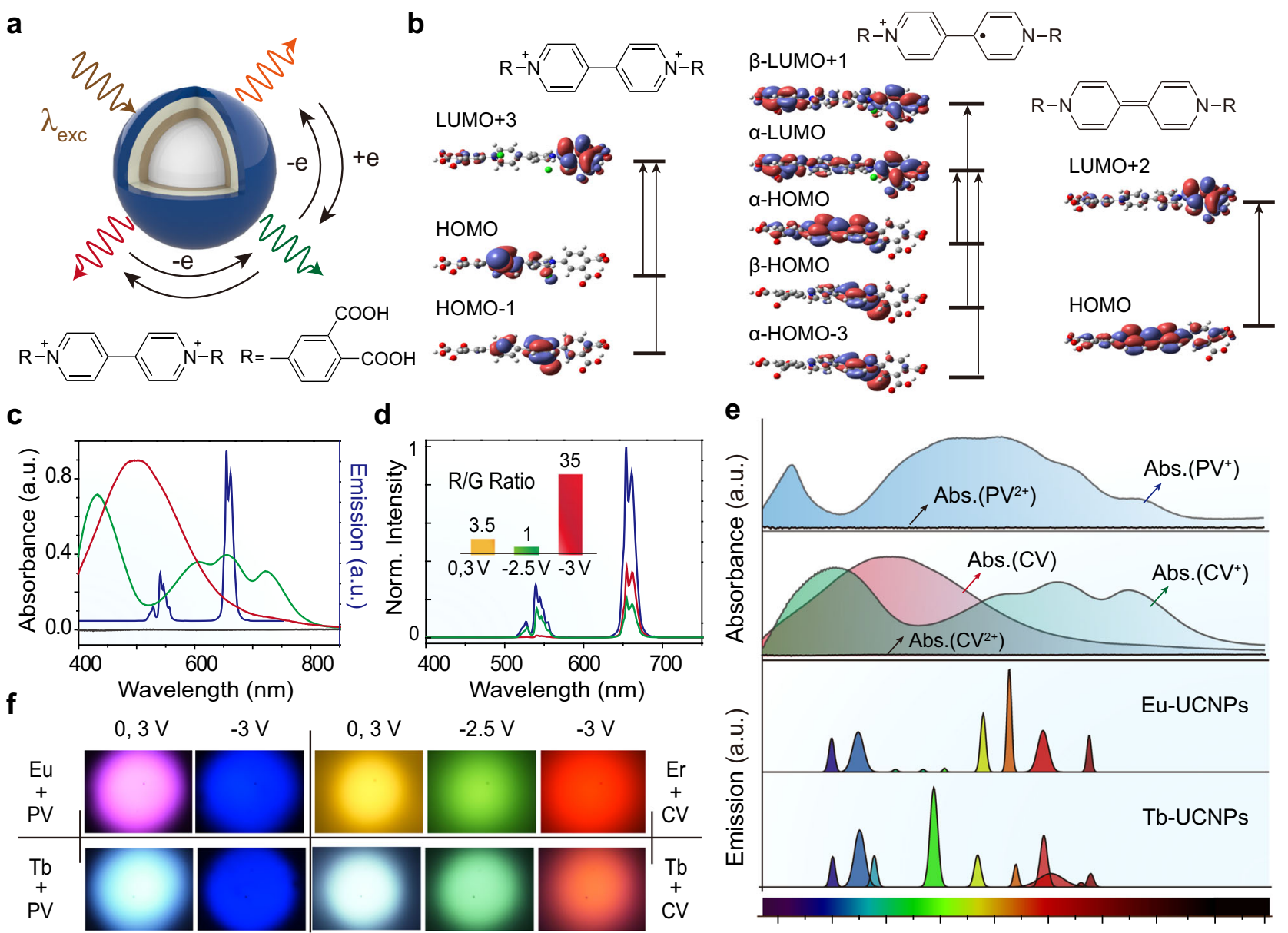

LUMO+3

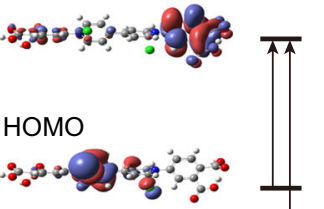

HOMO-1

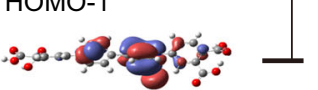

e

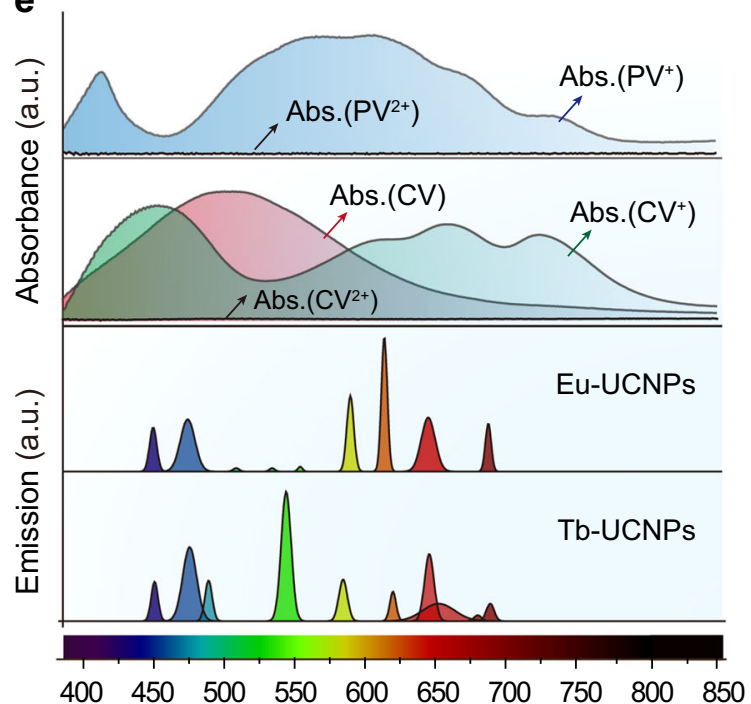

Fig. 3 Multicolour luminescence switching of UCNPs through opto-electrochemical modulation. a Schematic showing achievement of electrically multicolour switching using UCNPs modified with 1,1'-bis(3,4-dicarboxyphenyl)-4,4'-bipyridinium dichloride (CV) molecules. b DFT-calculated molecular orbital diagrams and dominant absorption transitions of 1,1'-bis(3,4-dicarboxyphenyl)-4,4'-bipyridinium dichloride molecule in three reduced and oxidised states. c Emission spectrum (blue) of the hybrid film and absorption spectra of CV molecules in the oxidised state (black), first reduced state (green), and second reduced state (red). d Emission spectra of the hybrid film under different potentials. Inset shows the red/green intensity ratios obtained under different potentials. e Absorption spectra of as-designed viologen molecules ( $P V$ and $C V$ ) in several reduced and oxidised states and deconvolution of emission spectra of two typical UCNPs ( $\mathrm{NaGdF}_{4}: \mathrm{Yb} / \mathrm{Tm}_{\mathrm{N}} @ \mathrm{NaYF}_{4}: \mathrm{Eu}$ and $\mathrm{NaGdF}_{4}: \mathrm{Yb} / \mathrm{Tm} @ \mathrm{NaYF}_{4}: \mathrm{Tb}$ ) into individual Gaussian peaks. f Upconversion multicolour switching of a wide variety of UCNP/Viologen systems under different potentials.

Upconversion multicolour editing. To further demonstrate the versatility of the MASET strategy to realise multicolour switching (Fig. 3a), we designed a molecule, 1,1'-bis(3,4-dicarboxybenzyl)4,4'-bipyridinium dichloride (CV), using density functional theory simulations (Table S1 and Supplementary Figs. 14-19). Theoretical predictions and experimental investigations revealed that this molecule features three distinct redox states $\left(\mathrm{CV}^{2+}, \mathrm{CV}^{+}\right.$, and $\left.\mathrm{CV}^{*}\right)$ under different applied voltages, yielding diverse tunable absorption profiles (Fig. 3b and Supplementary Figs. 20, 21). Electrochemical cells based on $\mathrm{CV}$ molecules and $\mathrm{NaYF}_{4}: \mathrm{Yb} / \mathrm{Er}$ nanoparticles displayed dominant yellow emission peaks at 540 and $654 \mathrm{~nm}$ upon $980 \mathrm{~nm}$ excitation, owing to negligible absorption of visible and NIR radiation by the CV molecule in the oxidised state $\left(\mathrm{CV}^{2+}\right)$. However, applying an electric field of $-2.5 \mathrm{~V}$ markedly suppressed the red emission, but with a slight effect on the green emission at $540 \mathrm{~nm}$. This can be attributed to spectral overlap between the red emission of $\mathrm{NaYF}_{4}: \mathrm{Yb} / \mathrm{Er}$ nanoparticles and the absorption band of $\mathrm{CV}$ molecules in the first reduced state $\left(\mathrm{CV}^{+}\right)$at $\sim 660 \mathrm{~nm}$. Further increasing the voltage to $-3 \mathrm{~V}$ leads to the conversion of $\mathrm{CV}$ molecules from the first $\left(\mathrm{CV}^{+\cdot}\right)$ to the second $\left(\mathrm{CV}^{*}\right)$ reduced state, which features absorption in the blue-green spectral range, but negligible absorption in the red region. As a result, the hybrid device showed red emission (Fig. 3c and Supplementary Fig. 22). These results suggest that by adjusting the voltage within the range of -3 to $3 \mathrm{~V}$, dynamic multicolour editing from yellow to green to red emission is possible using CV molecule-modified $\mathrm{NaYF}_{4}: \mathrm{Yb} / \mathrm{Er}$ nanoparticles through modulation of the ratio of red/green emission under different voltages. For example, a low voltage of $-2.5 \mathrm{~V}$ results in a red/green ratio of 1 , giving rise to green emission. Increasing the voltage to $-3 \mathrm{~V}$ yields intense red emission with a red/green ratio of 35 (Fig. 3d). Based on the MASET strategy, a wide range of colours can be realised at the single-particle level by coupling upconversion nanocrystals possessing tunable emission bands (e.g., $\mathrm{NaGdF}_{4}: \mathrm{Yb} / \mathrm{Tm} @ \mathrm{NaYF}_{4}$ : $\mathrm{Eu}$ and $\mathrm{NaGdF}_{4}: \mathrm{Yb} / \mathrm{Tm} @ \mathrm{NaYF}_{4}: \mathrm{Tb}$ ) with viologen molecules having variable absorption profiles (Fig. 3e, $\mathrm{f}$ and Supplementary Figs. 23-25).

Nonlinear logic switching circuits. The ability to electrically modulate anti-Stokes luminescence enables the development of multifunctional nonlinear optoelectronic devices. As a proof-ofconcept, electrochemical cells based on PV molecules and $\mathrm{NaGdF}_{4}$ : $\mathrm{Yb} / \mathrm{Tm} @ \mathrm{NaYF}_{4}: \mathrm{Eu}$ nanoparticles (Eu-UCNPs) were fabricated as opto-electrochemical logic gates for signal processing. In our design, 


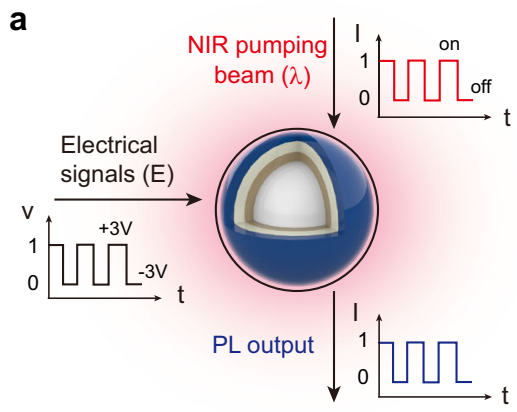

b

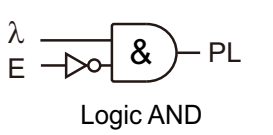

\begin{tabular}{cc|c}
$\ln 1$ & $\operatorname{In} 2$ & Out \\
$\lambda$ & $\mathrm{E}$ & $\mathrm{PL}$ \\
\hline 0 (off) & $0(-3 \mathrm{~V})$ & 0 \\
0 (off) & $1(+3 \mathrm{~V})$ & 0 \\
1 (on) & $0(-3 \mathrm{~V})$ & 0 \\
1 (on) & $1(+3 \mathrm{~V})$ & 1
\end{tabular}

d
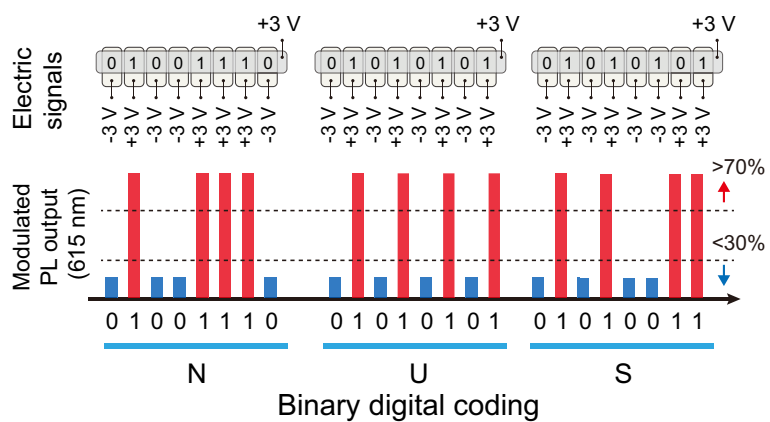

C

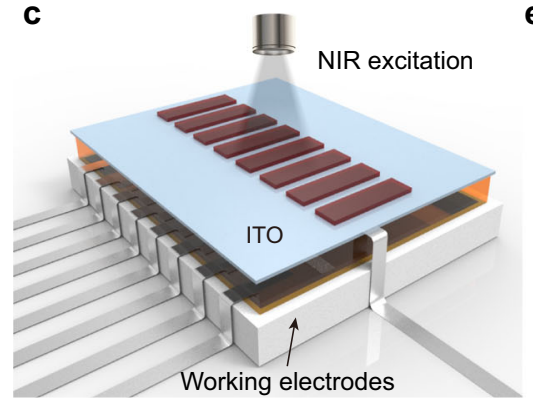

e $\quad$ : - - - - - - - - - - - - - - - - - - -

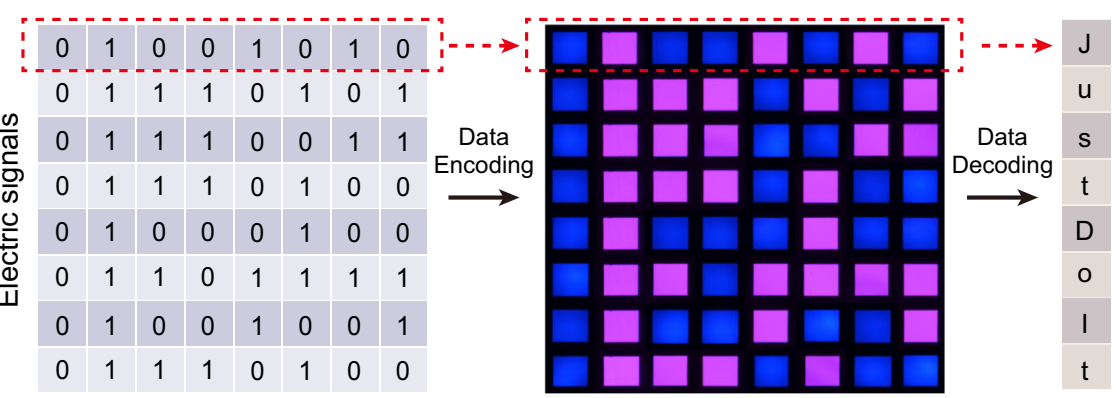

Fig. 4 Demonstration of opto-electrochemical logic AND gate using PV-modified UCNPs. a, b Basic operating principle of logic AND gate based on the Eu-UCNP/PV platform. The nonlinear output signal is programmed by a NIR laser and an alternating electric field. Under NIR illumination, the photonic readout is controlled by alternating the electric field, which initiates the redox reaction of PV molecules. c Schematic of an eight-pixeled, UCNP/PV logic gate array, in which each pixel is controlled individually by applying voltages of $\pm 3.0 \mathrm{~V}$. d Information processing of electrical inputs carrying the word "NUS" into optical signals using upconversion logic gating. The measured emission intensity of the Eu-UCNP/PV thin film at $615 \mathrm{~nm}$ below the $30 \%$ or above the $70 \%$ threshold represents binary codes, " 0 " or " 1 ", respectively. e Multiplexed data processing through opto-electrochemical modulation. A string of electrical signals carrying encrypted information of "Just Do It" is converted into visible patterns for signal output.

the $980 \mathrm{~nm}$ pumping beam (on or off) is considered the optical input (In1). Upon $980 \mathrm{~nm}$ excitation, Eu-UCNPs exhibit dominant blue and red emission peaks at 454 and $615 \mathrm{~nm}$, respectively. An alternating electric field (positive or negative) serves as the second input (In2), which can induce reversible spectral resonance between Eu-UCNPs and PV molecules. As such, an upconversion logic AND gate can be constructed with red emission as the output signal. Photonic readout signals, on (1) and off (0), are programmable by modulating NIR excitation and alternating electrical field (Fig. 4a, b).

We next demonstrated this principle by transmitting Roman letters using binary codes of standard eight-bit ASCII characters to digitally encoded optical outputs. Each letter is represented with an eight-digit binary code comprising different combinations of zeros and ones. For instance, the word "NUS" with three capital letters in a specific sequence is composed of data strings of 01001110, 01010101, and 01010011 in binary code. Figure 4c shows a schematic of the Eu-UCNPs/PV based logic gate array with eight pixels representing binary codes of standard eight-bit ASCII characters. Working electrodes (with UCNPs) and counter electrodes (ITO glass) are arranged orthogonally. Cross-sectional components are considered pixels of the matrix, which are controlled individually and independently. In our experimental setting, writing and erasing were performed by applying voltages of -3.0 and $+3.0 \mathrm{~V}$. When electrical signals carrying the word "NUS" were transmitted to the eight-pixeled UCNP/PV platform, the device converted electrical signals into upconverted emitting photons, encoding optical outputs of "NUS" in binary digits. Binary codes of " 0 " or " 1 " can be expressed by monitoring red emission intensity at $615 \mathrm{~nm}$ upon applying voltages of -3.0 and $+3.0 \mathrm{~V}$, respectively (Fig. 4d). Moreover, binary digital codes, " 0 " and "1", can also be represented with blue and red colours by applying alternating electrical pulses. We further demonstrated conversion of electrical signals "Just Do It" to high-fidelity photonic output through upconversion logic AND gate (Fig. 4e). Compared with DNA- and quantum dot-based switching circuits with readout based on changes in structural configuration or monochromatic emission intensity 39,40 , the multicolour switching characteristic of our system has advantages such as direct visualisation and easy identification of encrypted information.

\section{Discussion}

In summary, we have experimentally demonstrated precise control over anti-Stokes luminescence, based on coupling of lanthanide-activated nanoparticles and electrochemically responsive viologen molecules. By tailoring orbital energy levels of molecules under electric field modulation, the absorption profile of viologen molecules can be adjusted on-demand, enabling electrically tunable spectral resonance between UCNPs and dye species and ultimately resulting in dynamic multicolour editing of anti-Stokes luminescence. This molecularly engineered UCNP platform also enables opto-electrochemical signal processing for rapid, high-fidelity, and far-field communication. These results not only allow active control over anti-Stokes luminescence under low electric fields and pumping thresholds, but will also benefit the future development of nonlinear photonic devices for applications in quantum computing, multiplexed sensing, data encryption, and potentially many others.

\section{Methods}

Nanocrytal synthesis. Upconversion nanoparticles were synthesised using a coprecipitation method. Upconversion microrods were synthesised by a hydrothermal method. Detailed experimental procedures for the preparation of different types of nanoparticles/microrods are provided in the Supplementary Methods. 
Preparation of ligand-free $\mathbf{N a L n F}_{\mathbf{4}}$ nanocrystals. As-synthesised oleic acidcapped upconversion nanoparticles/microrods were dispersed in the mixture containing $1 \mathrm{~mL}$ of ethanol and $1 \mathrm{~mL}$ of $2-\mathrm{M} \mathrm{HCl}$ solution. The resulting mixture was ultrasonicated for $10 \mathrm{~min}$ to remove oleic acid ligands. Ligand-free nanoparticles were collected by centrifugation, washed with ethanol/deionized water several times, and re-dispersed in deionized water.

Preparation of $\mathbf{N a L n F}_{\mathbf{4}} @ \mathrm{SiO}_{\mathbf{2}} @ \mathrm{TiO}_{\mathbf{2}}$ nanoparticles. To a 50-mL round-bottom flask containing $15 \mathrm{~mL}$ of ethanol and $5 \mathrm{~mL}$ of deionized water were added $250-\mathrm{mg}$ PVP under stirring. After dissolving the PVP surfactant, ligand-free nanoparticles (0.2f poly(vinyl pyrrolidone) (PVP) mmol) were added. After stirring for $30 \mathrm{~min}$, a $300-\mu \mathrm{L}$ ethanol solution containing $50-\mu \mathrm{L}$ tetraethyl orthosilicate was added. The resulting mixture was reacted overnight under stirring. $\mathrm{NaLnF}_{4} @ \mathrm{SiO}_{2}$ nanoparticles were collected via centrifugation at $16,500 \mathrm{rpm}$ for $30 \mathrm{~min}$, followed by washing with ethanol twice. To a $50-\mathrm{mL}$ round-bottom flask containing $15 \mathrm{~mL}$ of methanol and $5 \mathrm{~mL}$ of deionized water were added 250-mg PVP under stirring. After dissolving the PVP surfactant, a solution of as-prepared silica-modified $\mathrm{NaLnF}_{4}$ core-shell nanoparticles was added. After stirring for $30 \mathrm{~min}$, a $200-\mu \mathrm{L}$ methanol solution containing titanium diisopropoxide bis(acetylacetonate) precursor was added. The resulting mixture was kept overnight under stirring. $\mathrm{NaLnF}_{4} @ \mathrm{SiO}_{2} @ \mathrm{TiO}_{2}$ nanoparticles were collected by centrifugation and washed with ethanol and deionized water several times.

Preparation of $\mathbf{N a L n F}_{\mathbf{4}} @ \mathrm{TiO}_{\mathbf{2}}$ microrods. Typically, a mixture solution containing $2 \mathrm{~mL}$ of PVP-40 $(0.2 \mathrm{~g} / \mathrm{mL})$ aqueous solution and $5 \mathrm{~mL}$ of ethanol was added $0.1 \mathrm{mmol}$ as-prepared ligand-free $\mathrm{NaLnF}_{4}$ microrods under stirring. A $2 \mathrm{~mL}$ freshprepared $\mathrm{TiF}_{4}(0.04 \mathrm{M})$ aqueous solution was dropwise added into the above solution. The mixture solution was then transferred into a Teflon-lined autoclave and heated to $180^{\circ} \mathrm{C}$ for $6 \mathrm{~h}$. The resulting products were collected by centrifugation and washed with ethanol and deionized water several times.

\section{Synthesis of 1,1'-bis(2-phosphonoethyl)-4,4'-bipyridinium dichloride. To a} $100-\mathrm{mL}$ round-bottom flask containing $50 \mathrm{~mL}$ of water were added 4,4'-bipyridine $(2.35 \mathrm{~g}, 15 \mathrm{mmol})$ and diethyl-2-bromoethyl phosphonate $(7.5 \mathrm{~g}, 30 \mathrm{mmol})$. Upon refluxing for $48 \mathrm{~h}, 8 \mathrm{~mL}$ of concentrated hydrochloric acid was added to the reaction mixture and refluxed for another $24 \mathrm{~h}$. After cooling to room temperature, the reaction mixture was concentrated to $5 \mathrm{~mL}$, followed by dropwise addition of 2 propanol. Upon stirring in an ice bath, the resulting products were filtered, washed with cold 2-propanol three times, and dried in a vacuum oven. ${ }^{1} \mathrm{H}$ NMR $(300 \mathrm{MHz}$, $\left.\mathrm{D}_{2} \mathrm{O}\right) \delta 9.08(\mathrm{~d}, J=6.3 \mathrm{~Hz}, 4 \mathrm{H}), 8.46(\mathrm{~d}, J=6.4 \mathrm{~Hz}, 4 \mathrm{H}), 4.85(\mathrm{dt}, J=12.4,7.8 \mathrm{~Hz}$, $4 \mathrm{H}), 2.38(\mathrm{dt}, J=17.5,7.7 \mathrm{~Hz}, 4 \mathrm{H})$

Synthesis of 1,1'-bis(3,4-dicarboxybenzyl)-4,4'-bipyridinium dichloride. To a 50 - $\mathrm{mL}$ flask charged with acetonitrile were added 4,4'-bipyridine $(2.5 \mathrm{~g}, 16 \mathrm{mmol})$ and 2,4-dinitro-chlorobenzene $(7.0 \mathrm{~g}, 34 \mathrm{mmol})$. The reaction mixture was refluxed at $80^{\circ} \mathrm{C}$ for $12 \mathrm{~h}$ under stirring. After reaction, the resulting 1,1'-bis(2,4-dinitrophenyl)bipyridinium dichloride was filtered and washed with acetonitrile three times and with diethyl ether four times, and dried in a vacuum oven. Compound 4aminophthalic acid in ethanol and a stoichiometric amount of 1,1'-bis(2,4-dinitrophenyl)-bipyridinium dichloride were added into the flask. The mixture was refluxed for $24 \mathrm{~h}$ under stirring. The solution was evaporated after filtration and extraction. The residue was dissolved in methanol and added to diethyl ether. Precipitated products were collected by filtration, washed with ethyl acetate/diethyl ether several times, and dried in a vacuum oven. ${ }^{1} \mathrm{H}$ NMR (500 MHz, DMSO) $\delta 9.79(\mathrm{~d}, J$ $=7.0 \mathrm{~Hz}, 4 \mathrm{H}), 9.16(\mathrm{~d}, J=7.1 \mathrm{~Hz}, 4 \mathrm{H}), 8.37(\mathrm{~d}, J=2.3 \mathrm{~Hz}, 2 \mathrm{H}), 8.21(\mathrm{dd}, J=8.3,2.4$ $\mathrm{Hz}, 2 \mathrm{H}), 8.09$ (d, $J=8.3 \mathrm{~Hz}, 2 \mathrm{H}) .{ }^{13} \mathrm{C}$ NMR (126 MHz, DMSO) $\delta 168.21,167.33$, $149.64,146.79,143.50,136.58,134.46,130.12,128.13,127.06,126.25$.

Fabrication of opto-electrochemical devices. In brief, the prepared $\mathrm{NaLnF}_{4} @$ $\mathrm{SiO}_{2} @ \mathrm{TiO}_{2}$ nanoparticles were mixed with a $\mathrm{TiO}_{2}$ paste, and the mixture was dispersed in ethanol containing $5 \mathrm{wt} \%$ PEG to form a stock slurry under stirring overnight. The fluorine-doped tin oxide (FTO) $\left(15 \Omega \mathrm{cm}^{-2}\right)$ glass was cleaned by oxygen plasma for $20 \mathrm{~min}$. A compact $\mathrm{TiO}_{2}$ layer was formed on the FTO glass by spin-coating the mixed solution $(0.1 \mathrm{M})$ of titanium diisopropoxide bis(acetylacetonate) in 1-butanol on the substrate and then annealed at $400{ }^{\circ} \mathrm{C}$ for $30 \mathrm{~min}$. The UCNPs@SiO $\mathrm{STiO}_{2}$ hybrid film was prepared on the $\mathrm{TiO}_{2}$-coated FTO glass by a doctor blade method. The hybrid film was then dried at room temperature for $30 \mathrm{~min}$ and then heated at $80^{\circ} \mathrm{C}$ for another $30 \mathrm{~min}$, followed by annealing at $400^{\circ}$ $\mathrm{C}$ for $20 \mathrm{~min}$ in a vacuum tube furnace. The hybrid film was immersed in a $50-\mathrm{mM}$ aqueous solution of viologen molecules for $12 \mathrm{~h}$. The substrate was washed with ethanol and then dried in a vacuum oven overnight. To construct the electrochemical cell, UCNPs@SiO $\mathrm{STiO}_{2}$ nanoparticle-based working electrode and another ITO counter electrode $\left(6 \Omega \mathrm{cm}^{-2}\right)$ were joined using double-sided tape. A solution of $0.5 \mathrm{M} \mathrm{LiClO}_{4}$ in propylene carbonate was injected as the electrolyte into the cell after removal of air.

Physical measurements. Powder X-ray diffraction data were obtained from a Bruker D8 Advance diffractometer using graphite-monochromatized CuKa radiation $(\lambda=1.5406 \AA)$. Transmission electron microscopy images were collected from a JEOL-JEM $2100 \mathrm{~F}$ electron microscope. Scanning electron microscopy images were taken on a JEOL-JSM-6701F electron microscope. ${ }^{1} \mathrm{H}$ and ${ }^{13} \mathrm{C}$ NMR spectra were recorded on a Bruker Advance 500 spectrometer at ambient temperature. UV-vis absorption spectra were recorded with a SHIMADZU ultraviolet3600 spectrophotometer. Electrochemical characterisation of the viologen molecule-modified electrode employed a CS310 potentiostat with a $\mathrm{Ag} / \mathrm{AgCl} / 1 \mathrm{M}$ $\mathrm{KCl}(\mathrm{aq})$ reference electrode.

Optical characterisations. Optical characterisations were carried out using the custom-built microscope capable of luminescence imaging and spectroscopy. For photoluminescence measurements, a $980 \mathrm{~nm}$ continuous-wave excitation laser is coupled to an optical microscope and focused onto the samples. The $980 \mathrm{~nm}$ excitation laser with the power intensity of $\sim 200 \mathrm{~W} / \mathrm{cm}^{2}$ was used for all the experiments. The photoluminescence is collected through a microscope objective and passed through an $800 \mathrm{~nm}$ short-pass fluorescence filter and sent onto a fibrecoupled Ocean Spectrometer.

\section{Data availability}

The authors declare that the data that support the findings of this study are available within the article and its Supplementary Information files. All other relevant data are available from the corresponding author upon reasonable request.

Received: 11 October 2020; Accepted: 27 January 2021; Published online: 01 April 2021

\section{References}

1. $\mathrm{Yu}, \mathrm{H}$. et al. High-gain infrared-to-visible upconversion light-emitting phototransistors. Nat. Photon. 10, 129-134 (2016).

2. Chen, $X$. et al. Confining energy migration in upconversion nanoparticles towards deep ultraviolet lasing. Nat. Commun. 7, 1-6 (2016).

3. Zhou, W. et al. Solution-processed upconversion photodetectors based on quantum dots. Nat. Electron. 3, 251-258 (2020).

4. Chen, S. et al. Near-infrared deep brain stimulation via upconversion nanoparticle-mediated optogenetics. Science 359, 679-684 (2018).

5. Lu, Y. et al. Tunable lifetime multiplexing using luminescent nanocrystals. Nat. Photon. 8, 32-36 (2014).

6. $\mathrm{Wu}, \mathrm{Y}$. et al. Upconversion superburst with sub-2 $\mu$ s lifetime. Nat. Nanotechnol. 14, 1110-1115 (2019).

7. Yan, C., Dadvand, A., Rosei, F. \& Perepichka, D. F. Near-IR photoresponse in new up-converting $\mathrm{CdSe} / \mathrm{NaYF}_{4}: \mathrm{Yb}, \mathrm{Er}$ nanoheterostructures. J. Am. Chem. Soc. 132, 8868-8869 (2010).

8. Tsang, M. K., Bai, G. \& Hao, J. Stimuli responsive upconversion luminescence nanomaterials and films for various applications. Chem. Soc. Rev. 44, 1585-1607 (2015).

9. Hernández-Rodríguez, M. A. et al. Lanthanide luminescence to mimic molecular logic and computing through physical inputs. Adv. Opt. Mater. 8, 2000312 (2020).

10. Cai, W., Vasudev, A. P. \& Brongersma, M. L. Electrically controlled nonlinear generation of light with plasmonics. Science 333, 1720-1723 (2011).

11. Seyler, K. L. et al. Electrical control of second-harmonic generation in a $\mathrm{WSe}_{2}$ monolayer transistor. Nat. Nanotechnol. 10, 407-411 (2015).

12. Yanai, N. \& Kimizuka, N. Stimuli-responsive molecular photon upconversion. Angew. Chem. Int. Ed. 59, 2-15 (2020).

13. Prigozhin, M. B. et al. Bright sub-20 nm cathodoluminescent nanoprobes for electron microscopy. Nat. Nanotechnol. 14, 420-425 (2019).

14. Wen, S. et al. Future and challenges for hybrid upconversion nanosystems. Nat. Photon. 13, 828-838 (2019).

15. $\mathrm{Gu}, \mathrm{Y}$. et al. High-sensitivity imaging of time-domain near-infrared light transducer. Nat. Photon. 13, 525-531 (2019).

16. Fan, Y. et al. Lifetime-engineered NIR-II nanoparticles unlock multiplexed in vivo imaging. Nat. Nanotechnol. 13, 941-946 (2018).

17. Deng, R. et al. Temporal full-colour tuning through non-steady-state upconversion. Nat. Nanotechnol. 10, 237-242 (2015).

18. Zhang, C. et al. Luminescence modulation of ordered upconversion nanopatterns by a photochromic diarylethene: rewritable optical storage with nondestructive readout. Adv. Mater. 22, 633-637 (2010).

19. Zhang, C. et al. White-light emission from an integrated upconversion nanostructure: toward multicolour displays modulated by laser power. Angew. Chem. Int. Ed. 54, 11531-11535 (2015).

20. Zhou, J. et al. Activation of the surface dark-layer to enhance upconversion in a thermal field. Nat. Photon. 12, 154 (2018).

21. Zou, H. et al. Thermal enhancement of upconversion by negative lattice expansion in orthorhombic $\mathrm{Yb}_{2} \mathrm{~W}_{3} \mathrm{O}_{12}$. Angew. Chem. Int. Ed. 58, 17255-17259 (2019). 
22. Hao, J., Zhang, Y. \& Wei, X. Electric-induced enhancement and modulation of upconversion photoluminescence in epitaxial $\mathrm{BaTiO}_{3}: \mathrm{Yb} / \mathrm{Er}$ thin films. Angew. Chem. Int. Ed. 50, 6876-6880 (2011).

23. Martínez, E. D. et al. Electrochromic switch devices mixing small- and large-sized upconverting nanocrystals. Adv. Funct. Mater. 29, 1807758 (2019).

24. Liu, Y., Wang, D., Shi, J., Peng, Q. \& Li, Y. Magnetic tuning of upconversion luminescence in lanthanide-doped bifunctional nanocrystals. Angew. Chem. Int. Ed. 52, 4366-4369 (2013).

25. Ye, S. et al. Modulated visible light upconversion for luminescence patterns in liquid crystal polymer networks loaded with upconverting nanoparticles. Adv. Opt. Mater. 5, 1600956 (2017).

26. $\mathrm{Xu}, \mathrm{T}$. et al. High-contrast and fast electrochromic switching enabled by plasmonics. Nat. Commun. 7, 1-6 (2016).

27. Wang, Y. et al. A multicolour bistable electronic shelf label based on intramolecular proton-coupled electron transfer. Nat. Mater. 18, 1335-1342 (2019).

28. Guo, Q. H. et al. Artificial molecular pump operating in response to electricity and light. J. Am. Chem. Soc. 142, 14443-14449 (2020).

29. Kortz, C., Hein, A., Ciobanu, M., Walder, L. \& Oesterschulze, E. Complementary hybrid electrodes for high contrast electrochromic devices with fast response. Nat. Commun. 0, 1-7 (2019).

30. Cantrill, S. J., Chichak, K. S., Peters, A. J. \& Stoddart, J. F. Nanoscale borromean rings. Acc. Chem. Res. 38, 1-9 (2005).

31. Zhou, B. et al. Constructing interfacial energy transfer for photon up- and down-conversion from lanthanides in a core-shell nanostructure. Angew. Chem. Int. Ed. 55, 12356-12360 (2016).

32. Gargas, D. J. et al. Engineering bright sub-10 $\mathrm{nm}$ upconverting nanocrystals for single-molecule imaging. Nat. Nanotechnol. 9, 300 (2014)

33. Fernandez-Bravo, A. et al. Ultralow-threshold, continuous-wave upconverting lasing from subwavelength plasmons. Nat. Mater. 18, 1172-1176 (2019).

34. Garfield, D. J. et al. Enrichment of molecular antenna triplets amplifies upconverting nanoparticle emission. Nat. Photon. 12, 402-407 (2018).

35. Vidotti, M. \& Torresi, S. I. Nanochromics: old materials, new structures and architectures for high performance devices. J. Braz. Chem. Soc. 19, 1248-1257 (2008).

36. Cummins, D. et al. Ultrafast electrochromic windows based on redoxchromophore modified nanostructured semiconducting and conducting films. J. Phys. Chem. B 104, 11449-11459 (2000).

37. Boyer, J. C., Carling, C. J., Gates, B. D. \& Branda, N. R. Two-way photoswitching using one type of near-infrared light, upconverting nanoparticles, and changing only the light intensity. J. Am. Chem. Soc. 132, 15766-15772 (2010).

38. Becker, K. et al. Electrical control of Förster energy transfer. Nat. Mater. 10, 777-781 (2006).

39. Zhang, Y. et al. DNA origami cryptography for secure communication. Nat. Commun. 10, 1-8 (2019).

40. Geiselmann, M., Marty, R., De Abajo, F. J. G. \& Quidant, R. Fast optical modulation of the fluorescence from a single nitrogen-vacancy centre. Nat. Phys. 9, 785-789 (2013).

\section{Acknowledgements}

This work is supported by the Singapore Ministry of Education (MOE2017-T2-2-110) Agency for Science, Technology and Research (A*STAR) (grant no. A1883c0011), National Research Foundation, Prime Minister's Office, Singapore under its Competitive Research Program (award no. NRF-CRP15-2015-03) and under the NRF Investigatorship program (award no. NRF-NRFI05-2019-0003), National Key R\&D Program of China (2019YFC1604605), and National Natural Science Foundation of China (21771135). We thank X. Xie. and Q. Gu. for technical assistance.

\section{Author contributions}

Y.W. and X.L. conceived and designed the experiments. X.L. supervised the project. Y.W., J.X. and J.H.X. synthesised upconversion nanocrystals and organic molecules. Y.W and J.H.X. conducted optical experiments and characterisations. X.Q. performed theoretical simulations. Y.W. and X.L. wrote the manuscript with input from all authors.

\section{Competing interests}

The authors declare no competing interests.

\section{Additional information}

Supplementary information The online version contains supplementary material available at https://doi.org/10.1038/s41467-021-22387-7.

Correspondence and requests for materials should be addressed to X.Q. or X.L.

Peer review information Nature Communications thanks Oscar Loureiro Malt and the other, anonymous, reviewer(s) for their contribution to the peer review of this work.

Reprints and permission information is available at http://www.nature.com/reprints

Publisher's note Springer Nature remains neutral with regard to jurisdictional claims in published maps and institutional affiliations.

(c) (i) Open Access This article is licensed under a Creative Commons Attribution 4.0 International License, which permits use, sharing, adaptation, distribution and reproduction in any medium or format, as long as you give appropriate credit to the original author(s) and the source, provide a link to the Creative Commons license, and indicate if changes were made. The images or other third party material in this article are included in the article's Creative Commons license, unless indicated otherwise in a credit line to the material. If material is not included in the article's Creative Commons license and your intended use is not permitted by statutory regulation or exceeds the permitted use, you will need to obtain permission directly from the copyright holder. To view a copy of this license, visit http://creativecommons.org/ licenses/by/4.0/.

(C) The Author(s) 2021 